\title{
In Situ Electrochemical Measurements in the Nanoaquarium
}

Nicholas M. Schneider ${ }^{1}$, Jeung Hun Park ${ }^{2}$, Joseph M. Grogan ${ }^{1}$, Suneel Kodambaka ${ }^{2}$, Daniel A. Steingart ${ }^{3}$, Frances M. Ross ${ }^{4}$, and Haim H. Bau ${ }^{1}$

1. Department of Mechanical Engineering and Applied Mechanics, University of Pennsylvania, Philadelphia, PA 19104, USA.

2. Department of Materials Science and Engineering, University of California - Los Angeles, Los Angeles, CA 90095, USA.

3. Department of Mechanical and Aerospace Engineering and the Andlinger Center for Energy and the Environment, Princeton University, Princeton, NJ 08544, USA.

4. IBM T. J. Watson Research Center, Yorktown Heights, NY 10598, USA.

Electrochemical processes play a pivotal role in nanoscale science, from nanomanufacturing to battery technologies. Macroscale electrochemical measurements rely on monitoring spatially-averaged quantities such as current as a function of potential. However, detailed observations of the evolution of morphology at the nanoscale provide information about the driving kinetic reactions and mechanisms and conditions that lead to potentially catastrophic phenomena such a dendrite formation in batteries. Using in situ liquid cell electron microscopy, we overcome the limitations of conventional spatially averaged measurements and directly correlate morphology with current and voltage $[1,2,3]$.

Measurements were carried out in our custom made liquid cell, the nanoaquarium [4], which is equipped with electrodes (Figure 1). We observed nucleation and growth in well-studied acidic copper and zinc electrolytes to validate the performance of our device. The integrated platinum electrodes were connected to a potentiostat to control and record the potential and current as functions of time during cyclic voltammetry $(\mathrm{CV})$ and galvanostatic sweeps. Simultaneous visualization of the evolving growth morphology was carried out at $300 \mathrm{kV}$ with a Hitachi H9000 TEM.

$\mathrm{CV}$ curves were generated for both the zinc and copper electrolytes and found to be similar to the ones obtained in macroscopic experiments, illustrating the validity of the experimental data obtained with the liquid cell. Additionally, galvanostatic (constant current) deposition and stripping were performed. Galvanostatic conditions allow us to relate the total charge passed into the system to the volume of metal deposited (for example, via $\mathrm{Cu}^{2+}+2 \mathrm{e}^{-} \rightarrow \mathrm{Cu}$ ). Figure 2 shows typical galvanostatic data obtained in an acidic copper sulfate solution by applying current as a square wave, alternating between deposition and stripping conditions. This data illustrates the consistency of the electrical performance between each cycle, showing a high level of reproducibility. Simultaneous imaging allows the synchronization of the electrical data with the microscopy images. Figure 3 shows one of the galvanostatic cycles in Figure $2 \mathrm{a}$ matched with stills from the video.

In conclusion, the nanoaquarium is well-suited for in situ electrochemistry. The ability to probe the system for both accurate electrical measurements and nanometer spatial resolution provides vital critical insights into the underlying reactions and mechanisms. The setup will be widely applicable for investigating morphology transformations such as the transition from compact to dendritic growth in a range of materials [5]. 


\section{References:}

[1] A. Radisic, P. M. Vereecken, J. B. Hannon, P. C. Searson, and F. M. Ross, Nano Letters, volume 6, no. 2 (2006), p. 238-242.

[2] N. de Jonge and F. M. Ross, Nature Nanotechnology, volume 6, no. 11 (2011), p. 695-704.

[3] J. M. Grogan, N. M. Schneider, F. M. Ross, and H. H. Bau, Journal of the Indian Institute of Science, volume 92, no. 2 (2012), p. 295-308.

[4] J. M. Grogan and H. H. Bau, Journal of Microelectromechanical Systems, volume 92, no. 4 (2010), p. 885-894.

[5] The authors acknowledge funding, in part, from the National Science Foundation, grants 1129722 and 1066573. Electron microscopy was performed at the IBM T. J. Watson Research Center with the valuable experimental contributions of Dr. Mark C. Reuter and Mr. Arthur Ellis of IBM.
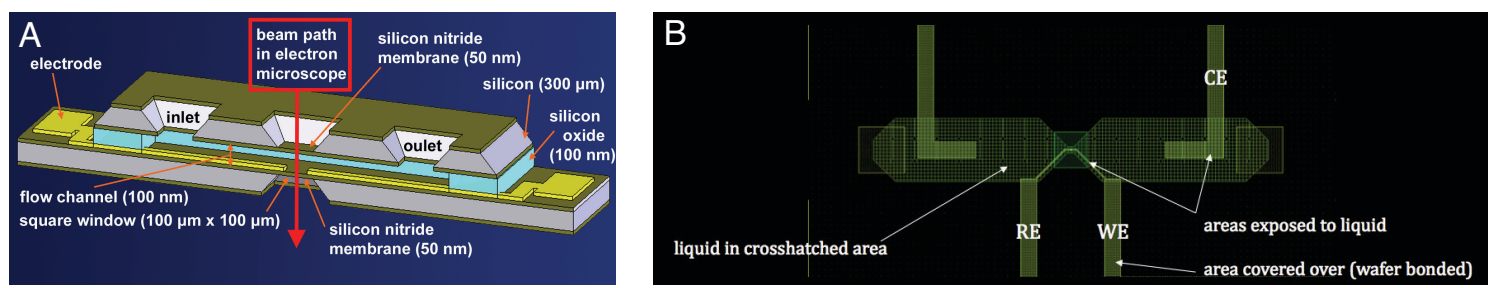

Figure 1: A) Cross-sectional view of the nanoaquarium. B) Electrode configuration used.
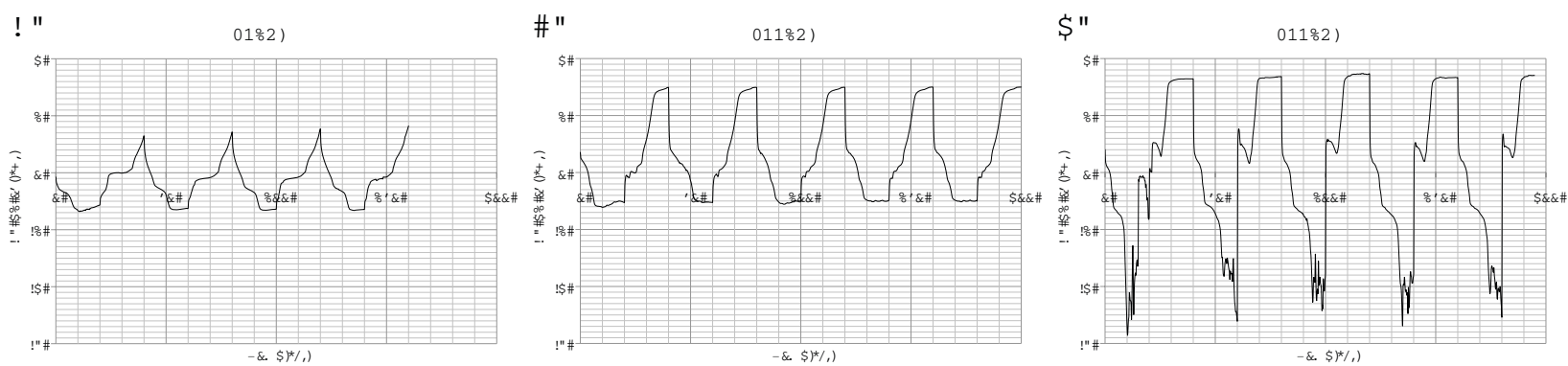

Figure 2: Working electrode potential as a function of time under galvanostatic conditions. Electrolyte solution of $0.1 \mathrm{M} \mathrm{CuSO}_{4}$ and $0.18 \mathrm{M} \mathrm{H}_{2} \mathrm{SO}_{4}$ in water. Square wave with alternating current of $\mathrm{A}$ ) $+/-50$ $\mathrm{nA} \mathrm{B})+/-100 \mathrm{nA} \mathrm{C})+/-200 \mathrm{nA}$ for repeated cycles of $20 \mathrm{~s}$ each.
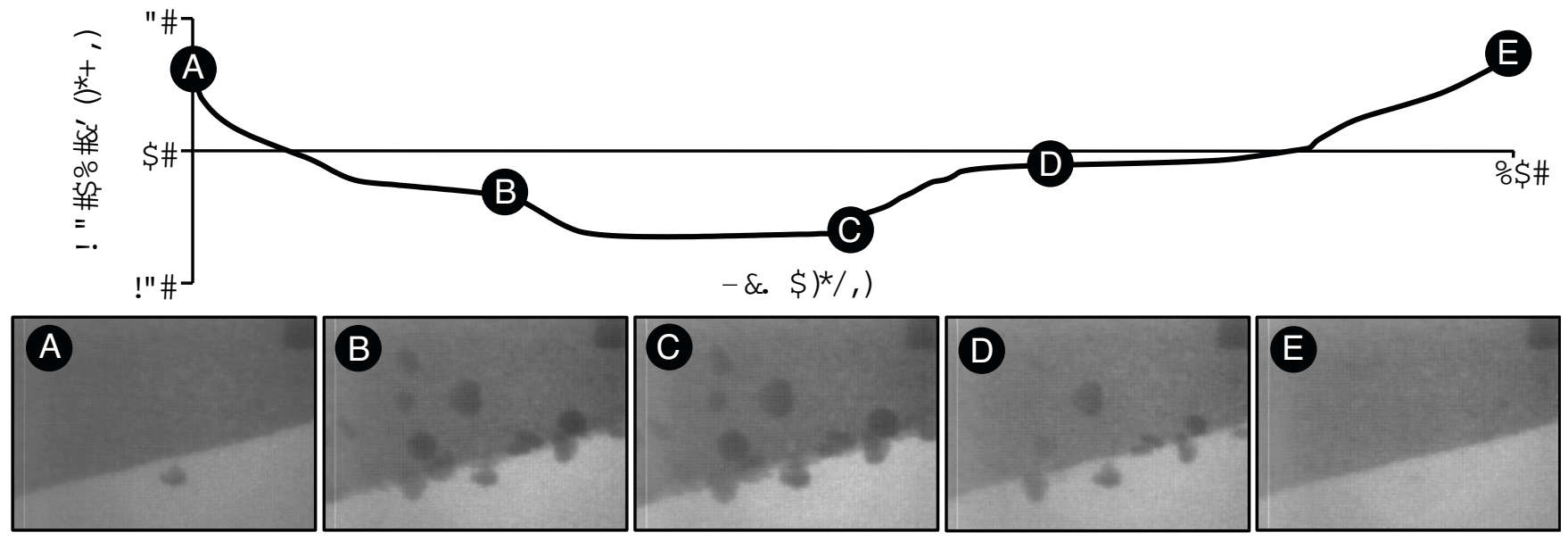

Figure 3: Second galvanostatic curve of Figure la for a square wave with alternating current of $-/+50$ $\mathrm{nA}$ with a period of $20 \mathrm{~s}$. Insets: TEM bright field images recorded at time of A) $0 \mathrm{~s}, \mathrm{~B}) 10 \mathrm{~s}, \mathrm{C}) 20 \mathrm{~s}$, D) $27 \mathrm{~s}$, and E) $40 \mathrm{~s}$. Each image is $1.9 \mu \mathrm{m}$ horizontally. 